\title{
Análisis comparativo del componente de práctica en el currículo de formación profesional de médicos y profesores en la Pontificia Universidad Católica de Chile*
}

\author{
Comparative Analysis of the Practical Component in the Curriculum of Professional Trai- \\ ning Health proffesionals and Teachers at Pontifical Catholic University of Chile
}

Análise comparativa do componente de prática no currículo de formação profissional de médicos e professores da Pontifícia Universidade Católica do Chile

\section{Sergio Martinic, ${ }^{a}$ Rodrigo Moreno, ${ }^{b}$ Magdalena Müller, ${ }^{c}$ Fernando Pimentel, ${ }^{d}$ Silvia Rittershaussen, ${ }^{e}$ Maribel Calderón ${ }^{f}$ y Héctor Cabezas ${ }^{g}$}

apUC, Facultad de Educación. Telf.: 56-2-23545312. Correo electrónico: smartini@uc.cl

bPUC, Facultad de Medicina. Director del Centro de Educación Médica. Telf.: 56-2-2354 3499. Correo electrónico: rmorenob@med.puc.cl

cPUC, Facultad de Educación. Telf.: 56-2-23545399. Correo electrónico: sritters@uc.cl

${ }^{d}$ PUC, Facultad de Medicina. Telf.: 56-2-2354 3499. Correo electrónico: fpimente@uc.cl

epUC, Facultad de Educación. Telf.: 56-2-23545399. Correo electrónico: mmullera@gmail.com

fPrograma de Doctorado en Psicología PUC. Telf.: 56-9- 88125703. Correo electrónico: mcalderon@uc.cl

gPrograma de Doctorado en Educación PUC. Telf.: 56-9-95029805. Correo electrónico: hacabezas@uc.cl

\section{RESUMEN}

Este artículo analiza la práctica profesional y clínica en el currículo de formación de Profesores de educación básica y de Médicos. Se analiza: i) las oportunidades de aprendizaje práctico en el currículo de ambas profesiones; ii) la organización del dispositivo pedagógico; iii) la relación de los cursos prácticos con el plan de estudios general; iv) la evaluación de las competencias adquiridas en los contextos prácticos o clínicos. Los resultados muestran diferencias en el dispositivo pedagógico, en el tiempo de duración y en los métodos de trabajo de los tutores y supervisores de prácticas. En este artículo se discuten dos temas claves para la formación de profesores: los vínculos entre el mundo académico y el campo de la práctica y la coherencia de los cursos curriculares generales con los diferentes niveles y experiencias prácticas.

Palabras clave: plan de estudios, práctica clínica, medicina, formación de profesores.

\begin{abstract}
This article reports the results of an analysis of the practice and clinical components in the training curriculum of basic education teachers and medical profession. It examines four aspects: i) opportunities for practical learning in the curriculum of both professions, ii) organization of practical pedagogical device, iii) relationship of the courses and clinical practices with the general curriculum, and iv) assessment of skills acquired in the practical or clinical contexts. The results show differences in the pedagogical device of the practices, in the length of the curriculum, and in the tutors and supervisors working methods. This paper analyzes and discusses two key issues about teacher training: the links between the academic and the practical field, and the need to articulate in a more coherent way the general curriculum courses with different levels of practice experiences.
\end{abstract}

Key words: curriculum, clinical practice field, medicine, basic education.

Patrocinado por la Vicerrectoría de Investigación y Doctorado. Programa especial de Investigación Interdisciplinaria en Educación. Pontificia Universidad Católica de Chile. 


\section{RESUMO}

Analisa-se a prática profissional e clínica no currículo de formação de Professores de Educação Básica e de Médicos. Analisa-se: i) oportunidades de aprendizagem prática no currículo de ambas as profissões; ii) a organização do aparato pedagógico; iii) a relação dos cursos práticos com o currículo geral e iv) a avaliação das competências adquiridas em contextos práticos ou clínicos. Resultados mostram diferenças nos aparatos pedagógicos, na duração e nos métodos de trabalho dos tutores e supervisores de práticas. Discutem-se dois temas essenciais para a formação de professores: a articulação entre o mundo acadêmico e o campo da prática e a coerência dos cursos curriculares gerais com os diferentes níveis e experiências práticas.

Palavras chave: currículo, campo de prática clínica, medicina, educação básica.

\section{INTRODUCCIÓN}

Existe un amplio consenso en la importancia del componente de práctica para mejorar la calidad de la formación profesional de profesores. La investigación reciente demuestra que gran parte del conocimiento pedagógico y profesional de los docentes se aprende a través de la práctica y de la observación de estas en contextos auténticos de desempeño profesional (Barber y Mourshed, 2008; Zeichner, 2010; Paquay, 2012).

La experiencia internacional ofrece distintos modelos que dan cuenta del nuevo status que asume la práctica formativa en el currículo de los futuros profesores. En los Estados Unidos, por ejemplo, se han expandido las Escuelas de Desarrollo Profesional que integran, desde los primeros años, a los futuros docentes a una práctica profesional con profesores expertos (Darling-Hammond, 2006, 2000). En la mayor parte de los países de la OCDE para obtener una licencia de profesor se exige un periodo de práctica docente obligatoria.

En Chile, se observan cambios similares que fueron animados, inicialmente, por el Proyecto de Fortalecimiento de la Formación Inicial Docente (FFID) del Ministerio de Educación (1997-2002). Uno de los principales logros informados por las 17 Facultades de Educación participantes fue la organización de experiencias progresivas de práctica profesional con vínculos fuertes entre las mismas universidades y los establecimientos del sistema educativo (Ávalos, 2002, 2007; Contreras et al., 2010).

La experiencia práctica en contextos auténticos de trabajo es una dimensión central para el aprendizaje de las habilidades, conocimientos y actitudes propias del futuro profesional docente. Por la importancia de esta práctica así como de la práctica clínica, diversos autores han relacionado la formación del profesor con la formación de los médicos (Schön, 1998; Shulman, 1998; Grossman, 2011).

En efecto, tanto en Medicina como en Educación, el aprendizaje en contexto o el conocimiento situado se ha señalado como fundamental para el aprendizaje del oficio (García, 2002; Marcelo, 2007; Contreras et al., 2010; Roget, 2009).

En Medicina la experiencia práctica ha sido una tradición en la historia de la Educación Médica. El aprendizaje de los principios conceptuales y prácticos del médico están estrechamente asociados al trabajo clínico en hospitales y consultorios donde el médico experto atiende sus pacientes en compañía del médico novato. Del mismo modo, en la historia de la formación de profesores, la práctica de los futuros docentes en contextos escolares ha sido un componente relevante en la formación docente desde las antiguas escuelas normales hasta las actuales escuelas universitarias.

Sin duda, hay grandes diferencias en las bases científicas, competencias y prácticas que enseñan ambas profesiones y que transcurren, a la vez, en tiempos curriculares muy diferentes. Pese a ello, en el campo de la educación, la formación médica suele ser un 
referente o modelo valorado en cuanto a la relación lograda entre teoría, práctica y docencia. Por ejemplo, un panel de expertos convocados en el año 2010 por el Ministerio de Educación, entre sus recomendaciones proponen se asigne recursos para la consolidación de redes de escuelas, liceos y colegios como campos de práctica profesional, muy en línea con lo que ocurre con la formación en medicina. A juicio de los expertos, la acreditación de las carreras de pedagogía debe estar condicionada a la existencia de una red de establecimientos que facilite la actividad de práctica profesional de los futuros profesores (Panel de Expertos, 2010).

La pregunta que se plantea es qué dimensiones de la experiencia práctica diseñada para el ejercicio de la formación de la medicina podrían ser comparadas, consideradas y aplicadas para perfeccionar la formación de los futuros profesores.

Para abordar este problema se realizó, en una primera fase, un análisis comparativo que diera cuenta de las bases conceptuales, metodológicas e institucionales que caracterizan la formación práctica contemplada en la oferta curricular de la formación de médicos y profesores de educación básica en la Pontificia Universidad Católica de Chile. Para ello, se consideraron focos o dimensiones orientadas a comprender lo que ocurre en el proceso de formación práctica. Estas refieren a qué es lo que se debe enseñar a través de las actividades prácticas; cómo se organiza el contexto y situación de práctica; cómo se realiza la enseñanza o cual es la metodología de la práctica y cómo se realiza o evalúan los resultados de la experiencia práctica.

En el presente artículo se reportan los resultados de la comparación de cuatro aspectos de la experiencia formativa. Estas son: oportunidades de aprendizaje práctico que contempla la oferta curricular; organización del dispositivo de práctica; relación de la práctica con el currículo y evaluación que se realiza de las competencias adquiridas a través de la práctica. Se presenta luego en la discusión una síntesis de las similitudes y diferencias encontradas que surgen como hallazgos importantes de considerar para establecer comparaciones que puedan ser útiles para la formación práctica en ambas carreras.

\section{MARCO DE REFERENCIA}

La importancia que asume la práctica como componente curricular de la formación profesional va acompañada de cambios en las teorías y metodologías que sustentan dicha formación. Hoy día la práctica en la formación se conceptualiza como una acción reflexiva mediada por la práctica y no como la mera aplicación de conocimientos adquiridos previamente (Cochran-Smith \& Lytle, 1999; Pérez-Gómez, 2010).

En educación médica se utiliza el concepto de aprendizaje en el contexto, que considera que los estudiantes aprenden más si lo hacen en el mismo contexto en el cual usarán los conocimientos (Dent, 2005; López \& Lázaro, 2010). En este sentido, la práctica clínica es el proceso de la actuación médica relacionada con la atención a la salud de un paciente. Sus componentes son la información clínica obtenida, las percepciones, los razonamientos, los juicios, los procedimientos utilizados, las decisiones y las intervenciones que se aplican (Munté, 2006).

En medicina, Caraccio y cols. (2008), siguiendo el modelo de Dreyfus y Dreyfus (1986), señalan que la adquisición de experticia en el razonamiento médico evoluciona en el tiempo mediante la experiencia de ver muchos pacientes de un tipo determinado. 
La expresión máxima de la pericia clínica se sitúa en el último de los cinco escalones del nivel de habilidades definidos por estos autores: novel, principiante avanzado, competente, diestro y experto.

En las escuelas de educación se habla de conocimiento situado y distribuido que refiere a un aprendizaje en diálogo con el entorno o ambiente con el cual el sujeto interactúa en su formación profesional (Marcelo García, 2002; Maroy, 2001; Contreras et al., 2010; Roget, 2009).

El currículo práctico considera las competencias y los contenidos que se espera sean aprendidos en los distintos cursos y niveles de práctica. Se abordan aspectos tales como la formulación de las competencias (como objetivos o competencias a alcanzar) y los referentes utilizados en su formulación, además de la proporción, secuenciación y progresión en el tiempo de las actividades de práctica y su articulación con el currículo de formación. La organización del dispositivo de práctica alude a la organización institucional de los diferentes aspectos necesarios de considerar para la realización de las actividades prácticas. Se aborda la identificación de los diferentes tipos de contexto, centros y situaciones en que se realizan prácticas, los criterios utilizados en su selección, la forma de acceso y el tiempo de permanencia en ellos. Considera, además, las actividades pedagógicas en la práctica, referidas a la relación que se establece entre docentes, estudiantes y conocimiento o competencias a aprender. Implica aspectos tales como los roles y funciones a desempeñar por docentes supervisores y tutores en la conducción de los procesos de aprendizaje de los alumnos y la caracterización de las diferentes tareas a ser desempeñadas por los alumnos en práctica.

La Relación de las prácticas con el currículo refiere a la consistencia del componente de práctica contemplando el currículo general de formación. Este foco de análisis puede permitir dar cuenta de la conceptualización de formación práctica que subyace en las propuestas, identificando su planteamiento respecto de la relación teoría práctica (Contreras et al., 2010).

La Evaluación alude al tipo de actividad que se realiza para verificar los aprendizajes en el proceso de práctica. Se abordan aspectos como los criterios, estándares e indicadores de evaluación y los procedimientos e instrumentos de evaluación implementados.

\section{MATERIAL Y MÉTODO}

Este es un estudio exploratorio y descriptivo y que ha seguido una estrategia cualitativa de investigación (Hernández et al., 2006; Babbie, 2000; Flick, 2004). Se distinguen tres fases: análisis documental; entrevistas docentes y entrevistas a estudiantes de niveles intermedios y avanzados de prácticas. En este artículo se resumen los principales resultados de la primera fase: el análisis documental.

El material de base para este análisis son los documentos y definiciones curriculares de ambas escuelas. Las principales fuentes documentales utilizadas son: i) perfiles de egresos declarados en los programas de ambas carreras; ii) estándares de desempeño propuestos por la Comisión Nacional de Acreditación tanto para Medicina como para Educación; iii) estándares de desempeño para la Formación Inicial Docente (MINEDUC, $2011)$; iv) programas de los cursos de práctica de Educación y de los cursos prácticos y de los internados en el caso de Medicina; y v) mallas curriculares de ambas carreras. 
Por otra parte, la experiencia académica del propio equipo de investigación en la gestión y docencia asociada a las prácticas ha permitido tener una visión selectiva y realista del funcionamiento de los dispositivos de prácticas.

Para el análisis de datos del material documental se ha aplicado un análisis de contenido. Este es uno de los procedimientos clásicos para analizar el material textual con independencia de su procedencia (Flick, 2004).

El análisis de contenido es un proceso teóricamente fundado cuyo objeto es el análisis de discurso expresado a través de textos. El análisis sigue un procedimiento que permite la construcción de categorías; la descripción y comparación de los textos y la interpretación de las relaciones internas y externas que tienen dichas categorías.

En el campo de la investigación cualitativa existen distintas estrategias para llevar a cabo el análisis de contenido. En este estudio hemos optado por seguir los principios de la "Grounded theory" (Glasser y Strauss, 1967; Strauss y Corbin, 1990).

Se definieron dominios y categorías al interior de los dominios lo que ayudó a la organización de la información obtenida de cada carrera y aseguró los criterios de comparación.

\section{RESULTADOS}

\subsection{OPORTUNIDADES DE APRENDIZAJE PRÁCTICO EN EL CURRÍCULO.}

\subsubsection{Perfiles de egreso}

El perfil profesional general de la carrera de Medicina de la Pontificia Universidad Católica de Chile (PUC), define las competencias que tienen que aprender sus egresados con bastante precisión. Ellas responden a consensos nacionales y a orientaciones internacionales de las escuelas de medicina.

En la definición del perfil se considera, además de las orientaciones de la propia Universidad, el perfil de competencias de los egresados de las carreras de Medicina de Chile, desarrollado por la Asociación de Facultades de Medicina de Chile (ASOFAMECH), en que describe 93 competencias agrupadas en 8 categorías; el perfil de egreso de la carrera de Medicina desarrollado por CNA para los procesos de acreditación y, por último, el perfil de Conocimientos del Examen Único de Conocimientos Médicos (EUNACOM), desarrollado por ASOFAMECH, en el que se describen con detalle los niveles de conocimiento que deben tener los egresados respecto a diagnóstico, tratamiento, seguimiento, exámenes de laboratorio y procedimientos, entre otros.

El egresado debe ser competente para atender problemas comunes de salud de la población chilena en el ámbito de la Cirugía, Medicina Interna, Obstetricia-Ginecología, Pediatría y Psiquiatría, entre otras. Se identifican las enfermedades que se deben conocer y todas las facultades deben garantizar su aprendizaje. Se definen estándares que actúan como una guía de lo que deben enseñar los profesores a nivel nacional y refieren, a la vez, a los contenidos que se evalúan en EUNACOM con un carácter obligatorio.

En Educación, en cambio, el proceso de definición de estándares es reciente. En el año 2001, y en el marco del programa de Fortalecimiento de la Formación Inicial, el Ministerio de Educación define por primera vez estándares de desempeño para la formación 
inicial. La formulación de estos consideró las opiniones de las Facultades de Educación, del Colegio de Profesores, de sostenedores, entre otros, y recogen gran parte de la experiencia internacional sobre la materia (Ávalos, 2002; Ministerio de Educación, 2001). Sin embargo, estos no tienen un carácter obligatorio y la evaluación de los mismos es algo reciente (Evaluación docente del Ministerio de Educación; ${ }^{1}$ Acreditación obligatoria de Pedagogías según Ley 20.129; examen INICIA). ${ }^{2}$

En el año 2011 se promulgan por primera vez Estándares Orientadores para Egresados de Carreras de Pedagogía en Educación Básica que incluyen estándares pedagógicos y disciplinarios para la enseñanza (MINEDUC, 2011). ${ }^{3}$

Por ello el currículo de la Facultad de Educación que es objeto de este análisis, y que fue definido en el año 2003, consideró los estándares como un referente, pero el resultado final no responde completamente a lo definido por el MINEDUC. En el currículo se definen tres grandes áreas de competencias profesionales. Estas son competencias en relación con el saber (6 competencias); en relación con el saber hacer (13 competencias) y en relación con el saber ser (7 competencias). En relación con el saber hacer, destacan: ser competente para seleccionar contenidos curriculares pertinentes para los estudiantes; formular y evaluar metas de aprendizajes; planificar y evaluar estrategias de enseñanza y de evaluación coherentes entre sí.

En la siguiente tabla se ejemplifican las competencias principales que se definen en el perfil de egreso de ambas carreras:

Tabla 1. Número y ejemplos de competencias en perfil de egreso en Medicina y en Educación ${ }^{4}$

\begin{tabular}{|l|c|}
\hline \multicolumn{1}{|c|}{ Medicina } & \multicolumn{1}{|c|}{ Educación } \\
\hline $\begin{array}{l}\text { a. Generales para desarrollar la práctica clínica. (23) } \\
\text { b. Para proveer atención médica de emergencia (5). }\end{array}$ & $\begin{array}{c}\text { a. Relación con el saber (6). } \\
\text { b. Relación con el saber } \\
\text { c. Para prescribir medicamentos (4). }\end{array}$ \\
$\begin{array}{l}\text { d. Para comunicarse en el ejercicio profesional (6). } \\
\text { e. Para realizar procedimientos diagnósticos y }\end{array}$ & $\begin{array}{c}\text { c. Relación con el saber ser } \\
\text { terapéuticos (32). }\end{array}$ \\
f. Para el uso de la evidencia en la práctica médica (3). \\
g. Para aplicar principios bioéticos y legales en la \\
práctica de la medicina (13). \\
h. Para trabajar efectivamente en los sistemas de salud \\
(7).
\end{tabular}

Desde el 2003 y sólo para los docentes que trabajan en el sistema municipal.

2 INICIA es una evaluación diagnóstica que rinden voluntariamente, desde el 2008, estudiantes egresados de Educación Básica y Educación de Párvulos.

3 Los estándares han sido elaborados de acuerdo a la nueva estructura del sistema escolar definida en la Ley General de Educación, promulgada en 2009, que establece que la Educación Básica consta de seis grados. Adicionalmente, los estándares asumen que el nuevo profesor generalista de la Educación Básica enseñará, fundamentalmente, en cuatro áreas disciplinarias: Lenguaje y Comunicación; Matemática; Historia, Geografía y Ciencias Sociales; y, Ciencias Naturales (Mineduc, 2011).

4 Lo números entre paréntesis indican la cantidad de competencias definidas al interior de las categorías señaladas en los perfiles de egreso de Medicina y de Educación. 
De este modo, tanto la Escuela de Medicina como la Facultad de Educación cuentan con estándares nacionales que definen las competencias a lograr a lo largo de la formación. Sin embargo, existen diferencias en cuanto al tiempo de aplicación; la obligatoriedad de los mismos y, particularmente, en cuanto a su definición y observación.

La escuela de Medicina, desde el origen de ASOFAMECH, en la década de los 60 asumió el perfil de egreso definido por las facultades que hasta entonces formaban a los médicos del país. En el año 1997 se definen los estándares a nivel nacional y todos los egresados rinden el EUNACOM que se aplica desde el año 2008 (Enriquez y Mena, 2005).

En educación la definición de perfiles de egreso y de competencias es reciente y solo en el 2011 se han promulgado estándares de formación que tienen aun un carácter orientador. La prueba INICIA es voluntaria y se discute actualmente en el parlamento un proyecto de ley de carrera docente que le otorga un carácter obligatorio.

Como se señaló, los estándares de desempeño para la formación inicial de profesores a diferencia de los de Medicina son: i) genéricos, es decir, describen acciones aplicables en distintos niveles escolares y en distintas especialidades; ii) se expresan en términos de forma de desempeño y no como una conducta específica.

En Medicina, en cambio, estas competencias se definen como la capacidad para desempeñar apropiadamente un conjunto de tareas complejas que requieren de la integración de conocimientos, habilidades y actitudes. Por ejemplo, el egresado debe "Renovar permanentemente los conocimientos efectuando preguntas relevantes, definiendo los objetivos de autoaprendizaje, buscando en forma eficiente la información apropiada para resolverlas, interpretando críticamente los resultados, juzgando si el conocimiento se aplica a las circunstancias clínicas, integrándolos a la práctica y evaluando los resultados". Debido a esta forma de definir sus competencias de egreso, Medicina cuenta con un conjunto de competencias específicas definidas con precisión, alineadas con los estándares nacionales e internacionales, las que permiten guiar en forma integrada los contenidos de los cursos, las actividades prácticas de los estudiantes y la evaluación sus aprendizajes.

Educación, por su parte, cuenta con competencias definidas en forma más general, que deben ser interpretadas por los profesores de cada curso afectando, en algunos casos, el alineamiento o coherencia buscada (Facultad de Educación, 2003).

En síntesis, se constatan diferencias en el modo de definir competencias en los perfiles de egreso y en la relación que estos tienen con las definiciones de competencias o estándares nacionales a lograr en la formación. A modo de hipótesis, las diferencias observadas en el nivel de especificidad de las competencias de los perfiles de egreso de ambas carreras se relacionan con el contexto de desempeño del futuro profesional. En Educación la enseñanza se percibe como una actividad altamente dependiente del contexto y, por lo tanto, más difícil de estandarizar, a diferencia de Medicina que se reconoce como una profesión con un alto nivel de estandarización y menos dependiente del contexto de desempeño.

\subsubsection{La progresión del aprendizaje de la práctica en el currículo}

En ambas carreras el currículo plantea un aprendizaje práctico progresivo. Las experiencias prácticas en los primeros años son menores y, por lo general, están asociadas a aplicaciones o a ejercicios en el marco de los cursos de base y en contextos que representan o que describen desempeños profesionales específicos. A medida que se progresa 
en la formación se avanza también en autonomía y ejercicio profesional en contextos auténticos (Grossman, Compton, Igra, \& Ronfeldt, 2011).

En Medicina, el aprendizaje práctico con pacientes ocupa aproximadamente el 54\% del tiempo programado para el total de la carrera (418 de 773 créditos). En el $46 \%$ restante, también hay actividades prácticas, en que los estudiantes desarrollan diferentes habilidades, como reconocimiento de estructuras anatómicas, lectura crítica y resolución de problemas. Una alta proporción de la actividad práctica se efectúa en los Internados de sexto y séptimo años, en los que los estudiantes efectúan actividades prácticas 60 horas por semana, incluyendo turnos de noche, fines de semana y festivos.

En Educación la línea de práctica progresiva representa el 12\% del currículo de formación del profesor de Educación Básica sin mención (50 de un total de 410 créditos). En el resto de los cursos del currículo también se realizan actividades de aprendizaje práctico alguno de los cuales implican ejercicio de competencias en establecimientos educativos.

En Medicina, en los primeros dos años, lo fundamental es la adquisición de los conocimientos de las disciplinas básicas y fundantes de la carrera, así como el inicio del desarrollo de las competencias profesionales generales.

En el tercer año los principales objetivos son el aprendizaje de las ciencias relacionadas con la enfermedad en general (Patología y Microbiología) y su tratamiento (Farmacología), así como la adquisición de las competencias clínicas elementales (comunicación, historia clínica, examen físico). En el siguiente ciclo, de cuarto y quinto años, los estudiantes aprenden las enfermedades específicas y desarrollan sus habilidades clínicas en cursos que mezclan la teoría y la práctica.

En Educación, en los dos primeros años predominan los cursos de formación básica y en el tercer y cuarto año los cursos del área de formación profesional. En forma paralela a las materias del currículo se realiza una práctica progresiva que contempla 4 niveles o cursos a partir del segundo año de la carrera.

El área de formación básica enseña el marco conceptual para comprender y abordar el fenómeno de la acción educativa. El área de formación profesional contiene materias orientadas al desarrollo de la identidad profesional docente; saberes disciplinarios y las didácticas. Los cursos de prácticas se articulan en torno a 3 ejes: autonomía y responsabilidad en la toma de decisiones; construcción de conocimientos e identidad profesional.

En ambos casos se define, a través de las prácticas, un acercamiento progresivo a la situación real de ejercicio profesional. En esta progresión el estudiante va adquiriendo mayor autonomía; realiza desempeños profesionales supervisados hasta llegar a un nivel de plena autonomía realizando actividad profesional en forma completa en distintos periodos de tiempo.

En el currículo analizado de la Facultad de Educación la línea de práctica progresiva contempla cuatro cursos organizados en torno a tres ejes que articulan integrada y progresivamente el proceso de formación. Estos son: autonomía y responsabilidad en la toma de decisiones; construcción de conocimientos e identidad profesional.

En las prácticas iniciales (I y II) el estudiante se acerca a los establecimientos principalmente como observador. El objetivo es lograr el desarrollo de capacidades de comprensión y análisis de las instituciones escolares; sus actores y específicamente del educando. Las prácticas están orientadas principalmente a fortalecer la vocación docente.

En el nivel III los estudiantes se integran a un centro educacional, bajo la tuición de un profesor colaborador con el fin de diseñar y experimentar propuestas acotadas de intervención 
didáctica supervisada. Por último en el nivel IV los estudiantes asisten a un establecimiento durante 5 jornadas escolares a la semana y asumen el rol profesional en forma autónoma y auténtica. La actividad se complementa con sesiones de reflexión de la experiencia práctica, sobre la base de núcleos de problematización (un módulo a la semana).

En Medicina la progresión se define en función de las competencias declaradas en el perfil de egreso. En la formación práctica clínica (que involucra pacientes reales o simulados), hay un entrenamiento progresivo que empieza en el primer semestre del tercer año y termina en el séptimo año. Inicialmente, los estudiantes aprenden a efectuar las subrutinas de la historia clínica del paciente y del examen físico, para seguir con su obtención sistemática en pacientes reales, luego aprenden a interpretar los hallazgos para formular hipótesis diagnósticas y a comprobarlas mediante exámenes de laboratorio, a formular planes de tratamiento y de seguimiento.

Finalmente, en los internados de sexto y séptimo años los internos practican la medicina bajo supervisión, como integrantes de los equipos de trabajo. El entrenamiento descrito se realiza en diferentes disciplinas (Medicina Interna, Medicina Familiar, Pediatría, Cirugía, Ginecología Obstetricia, Psiquiatría y otras especialidades (Dermatología, Oftalmología, Otorrino), así como también en diferentes ámbitos de atención (ambulatorio, intrahospitalario, urgencias) y en diferentes tipos de instituciones (públicas-privadas). Además de las competencias clínicas recién mencionadas, los estudiantes adquieren otras como lectura crítica o análisis ético, también de forma progresiva.

En síntesis, en ambas carreras existe una organización gradual o progresiva de las actividades de práctica, en términos de tiempo de dedicación, complejidad de las tareas a realizar y de la autonomía en su desempeño. En esta progresión el estudiante va adquiriendo mayor autonomía; partiendo con desempeños profesionales parciales contemplando apoyo tutorial permanente hasta llegar a niveles de desempeño de actividades profesionales complejas en distintos contextos con una supervisión moderada. Sin embargo, existen importantes diferencias en la práctica progresiva.

La primera de ellas tiene relación con la cantidad y forma de distribución de la carga horaria de práctica en el proceso de formación durante todos los años de las carreras. Si bien en ambas carreras hay progresión, en la formación médica el trabajo supervisado de atención de pacientes hacia el final se concibe como proceso único de formación, aumentando de manera exponencial. Esta situación formativa no ocurre en educación, donde la participación en escuelas si bien es mayor, en el último año no supera las 400 horas.

Gráfico 1. Distribución de horas según actividades prácticas en Medicina y Educación

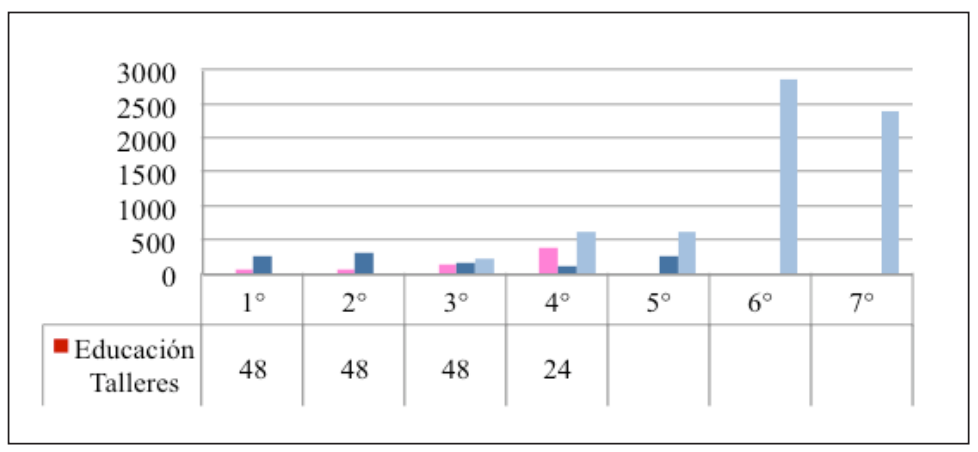


En segundo lugar, se diferencian ambas carreras en el concepto de competencias. En Medicina están definidas las competencias como integración de conocimientos, habilidades y actitudes ya validadas a realizar en una situación o problema determinado. La evidencia práctica y académica señala que frente a un determinado diagnóstico, hay cursos de acción ya comprobados como válidos para una situación similar. El estudiante investiga las bases de dichos procedimientos; observa su aplicación y los ejecuta en forma práctica.

En tercer lugar, tanto en Medicina como en Educación es muy importante que el estudiante egrese con la capacidad de aplicar el razonamiento pedagógico o médico en contextos profesionales reales. La formación del razonamiento pedagógico y del juicio médico es un proceso cognitivo que integra el aprendizaje teórico, la experiencia práctica y la propia madurez del estudiante. Para este proceso, en ambas carreras, son muy importantes la observación y aprendizaje de buenos modelos prácticos y el tiempo de maduración reflexivo-práctico del estudiante (Carraccio, Benson, Nixon, \& Derstine, 2008).

Por ello, el tiempo de duración de la carrera es un factor clave de diferenciación. Educación es una carrera relativamente corta ( 4 años +1 año de mención optativo) y no está garantizado que, al egresar, el estudiante haya desarrollado un razonamiento pedagógico con la madurez y seguridad deseada. Los estudiantes cuando realizan la práctica profesional, solo trabajan una unidad o dimensión del ejercicio profesional en un contexto protegido y de formación. El aprendizaje y la reflexión lo hacen como alumnos y no como un profesional propiamente tal. En las prácticas no viven la lógica de ser profesor ni tampoco asumen todas las acciones que, en forma simultánea, realizan los profesores en un establecimiento.

Medicina, en cambio, es una carrera más larga (7 años) y en los últimos dos años la formación está centrada en experiencia clínica profesional. En el internado los estudiantes deben resolver en forma completa supervisada problemas o situaciones planteados en el contexto del tratamiento de una enfermedad con pacientes reales. El internado es fundamental, indispensable, para la maduración del razonamiento médico.

Lo anterior plantea un problema más de fondo relacionado con los aprendizajes y maduración del razonamiento médico o pedagógico. Este no es lineal. En medicina inicialmente es muy lento pero, posteriormente, se progresa exponencialmente. El aprendizaje y desarrollo del razonamiento profesional no es una mera acumulación de conocimiento sino que, como señala Caraccio y cols. (2008) es una transformación evolutiva de conocimientos.

\subsection{LA ORGANIZACIÓN DEL DISPOSITIVO PEDAGÓGICO DE PRÁCTICA}

\subsubsection{Los centros de prácticas}

Existen claras diferencias entre las dos carreras en la organización de los dispositivos de prácticas. Ello se expresa fundamentalmente en el vínculo que se establece con los centros o campos de práctica, los criterios de selección, el acceso de los estudiantes y los tiempos de permanencia en cada centro de práctica a lo largo de la formación.

En Medicina los campos clínicos abarcan tanto establecimientos hospitalarios como ambulatorios, los que a su vez, pueden ser de atención de urgencias, de atención primaria (al que los pacientes concurren por enfermedades simples atendidas por médicos generales) y de atención secundaria (en que los pacientes son atendidos ambulatoriamente por 
especialistas). Los establecimientos hospitalarios o ambulatorios, son privados, de propiedad de la PUC, o públicos, gestionados por el Ministerio de Salud o por las municipalidades. Prácticamente todos los profesores a cargo de las prácticas de los estudiantes de medicina tienen una relación contractual con la PUC. En los establecimientos públicos, los médicos de dichos centros son nombrados profesores y contratados por la PUC.

Además, existen los Centros de Salud Familiar, Ancora, que se han formado a través de donaciones y son equipados por la PUC. A este se incorporan los estudiantes de pre y post grado, como también es un lugar de práctica para alumnos de psicología y enfermería.

Los centros asistenciales públicos han sido seleccionados por mutua conveniencia. Existen acuerdos que incluyen construcción o habilitación de espacios para la docencia, entrega de servicios a los pacientes y aportes por parte de la universidad. En estos espacios también se espera que se haga lo que la escuela y la formación definen. Ello se explicita a través de convenios. El acceso a los centros de prácticas está regulado por el Ministerio de Salud.

La Escuela de Medicina asegura a los estudiantes rotaciones por todos los contextos descritos previamente (disciplinas, especialidades, ámbitos de atención y tipos de instituciones) con el fin de lograr que todos adquieran una experiencia práctica similar en cada uno de los centros.

En Educación la situación es diferente. No existen establecimientos propios similares al campo clínico hospitalario. La Facultad establece convenios de colaboración con establecimientos educativos que son seleccionados en base a criterios de calidad y que corresponden a los tres tipos de dependencia que tiene el sistema educativo nacional. Los lugares de práctica son establecimientos municipales $(5 \%)$, particulares subvencionados $(80 \%)$ y particulares pagados $(14 \%)$. Los cursos en que se realiza la práctica son principalmente de $1^{\circ}$ a $6^{\circ}$ básico para las prácticas II, III y IV y para las prácticas de mención se trabaja en los cursos de $5^{\circ}$ a $8^{\circ}$. No existe acreditación de los establecimientos como Centros de Prácticas ni tampoco transferencia de recursos por su participación.

Las prácticas tienen una duración semestral y difieren en cuanto al tiempo de permanencia y de participación en la escuela. En las prácticas I y II los profesores en formación asisten una mañana al establecimiento, en práctica III dos mañanas y en la práctica IV asisten de lunes a viernes durante las mañanas. Por tratarse de centros de distintas características y por no contar con una relación formal entre establecimientos o profesores colaboradores y la Facultad, las experiencias de los estudiantes de Educación Básica pueden ser muy heterogéneas, con escaso control respecto a la generación de oportunidades de aprendizaje en la práctica en aspectos más específicos.

\subsubsection{Los formadores involucrados en la experiencia práctica}

En Medicina cada asignatura está a cargo de uno o dos profesores. Ellos, de acuerdo al programa concordado con el Director de Pregrado, diseñan y coordinan las actividades teóricas y prácticas, proveyendo los recursos materiales y humanos necesarios para las actividades programadas. Según la complejidad de las actividades prácticas, nombran encargados de coordinar las actividades docentes de una asignatura en los diferentes centros. En los grandes hospitales públicos existe además un académico nombrado por la Escuela, que efectúa una coordinación general de la docencia de pre y postgrado. 
Los profesores que trabajan directamente con los estudiantes son profesores de la Escuela de Medicina, que son seleccionados por los jefes de los departamentos respectivos de acuerdo a sus necesidades académicas y asistenciales. Prácticamente todos son médicos, con formación de postítulo en una especialidad de la medicina. Una proporción alta de ellos tiene formación de postgrado en el extranjero. La formación en docencia médica es más variada. Desde el año 2001, el Centro de Educación Médica viene efectuando un Diplomado en Educación Médica, del cual han egresado alrededor de 200 académicos. La mayor parte de los 800 académicos de la Escuela de Medicina han efectuado al menos un curso de este Diploma. Los profesores que participan en las actividades prácticas conocen el currículo así como los objetivos planteados para esas actividades. El profesor encargado se reúne con ellos antes del inicio de cada curso para aclarar modificaciones y novedades. El rol del profesor tutor considera la demostración, monitoreo, acompañamiento y evaluación del estudiante durante todo el tiempo que dura la práctica.

En Educación el trabajo directo con los estudiantes en práctica lo realizan los supervisores y los profesores colaboradores. El supervisor es un profesor con experiencia en trabajo en sala, que está a cargo de vincular a los estudiantes con los centros de práctica y establecer los requerimientos necesarios para generar el espacio para el practicante, en un ámbito administrativo. Su labor pedagógica incluye la supervisión de una cierta cantidad de clases a los estudiantes, monitorear el cumplimiento de las actividades requeridas en la práctica y estimular la reflexión grupal sobre el sentido de las experiencias pedagógicas en reuniones semanales. Los profesores supervisores son contratados por la Facultad de Educación de acuerdo al número de estudiantes que tengan a su cargo, incluyendo tiempo de acompañamiento directo en la escuela y tiempo de talleres semanales en la Universidad, además de reuniones de coordinación entre supervisores.

Los profesores colaboradores son los profesores a cargo de los cursos en las escuelas y colegios, quienes tienen el rol de ser modelos y guiar a estudiantes de pedagogía en formación. Estos profesores no tienen ninguna relación contractual con la Facultad de Educación y reciben voluntariamente a los estudiantes. Los profesores colaboradores trabajan con mucha autonomía y, por ello, las experiencias de práctica no son equivalentes para los estudiantes ya que dependen, principalmente, del profesor colaborador y del establecimiento asignado.

Los profesores supervisores en su mayoría conocen los programas y el currículo, sin embargo, los profesores colaboradores sólo están familiarizados con los lineamientos curriculares de las prácticas, no se les pide conocimiento del programa ni del currículo de formación general. Ambos actores, a su vez, no tienen relación directa con los profesores de cursos teóricos, y por lo general, tampoco con los cursos de didácticas.

\subsection{RELACIÓN DE LAS PRÁCTICAS CON EL CURRÍCULO}

En ambas carreras el contenido profesional se aprende fundamentalmente en la práctica. Cómo se ha señalado previamente en educación médica se trabaja bajo el concepto de aprendizaje en contexto y en educación sobre conocimiento situado.

Pese a la aparente cercanía de esta definición, se observan diferencias importantes en la forma de entender la relación teoría y práctica y en la articulación del componente de práctica con el resto del currículo.

En Medicina, los aprendizajes teóricos y prácticos se planifican coordinadamente en el currículo. Si bien existen cursos con objetivos completamente teóricos, especialmente 
en las asignaturas básicas de primero a tercer año, durante las actividades prácticas se revisa cada uno de los contenidos teóricos involucrados. Los cursos prácticos aplican los contenidos teóricos que se entregan en asignaturas diferentes o, paralelamente, en la misma asignatura cuando estas combinan actividades teóricas y prácticas.

Además, cuando un estudiante ve un paciente con una enfermedad determinada, debe efectuar una revisión teórica de la enfermedad del paciente y presentarla oralmente o en un portafolio, independientemente si ha aprendido o no los contenidos teóricos en los cursos. Los internos deben presentar todos los días los pacientes ingresados el día anterior, lo que incluye ser capaz de responder a las preguntas relacionadas con la enfermedad de los pacientes, para lo cual hacen revisiones bibliográficas.

En Educación, desde el punto de vista conceptual, existe una estrecha relación entre la línea de práctica progresiva y los cursos teóricos. Las competencias de egreso sólo pueden ser aprendidas y desarrolladas progresivamente en una articulación continua entre las teorías de la educación y la práctica del ejercicio profesional (Facultad de Educación, 2003: 27). Sin embargo, en la actual organización curricular la práctica progresiva constituye una línea paralela a los cursos del currículo. Cada actividad curricular de práctica tiene objetivos, contenidos y métodos propios para el nivel y diferentes a los cursos del currículo. Esto afecta la posibilidad de que las prácticas sean una oportunidad de aplicar lo aprendido en un curso teórico.

La separación entre cursos teóricos de la malla y prácticas profesionales se hace más compleja por la intervención de diferentes actores que, a la vez, tienen distintas relaciones contractuales y oportunidades de coordinación y trabajo conjunto. Existen así profesores de cursos de malla curricular, supervisores de prácticas y profesores colaboradores en los establecimientos. En la relación entre estos actores suelen ocurrir problemas de alineamiento; de coordinación y comunicación durante el seguimiento de los estudiantes.

\subsection{LA EVALUACIÓN}

En ambas carreras se espera que, a través de las prácticas, los estudiantes aprendan las competencias fundamentales para el desempeño de la profesión. En Medicina estas se refieren al aprendizaje de competencias en todas las especialidades y ámbitos de atención en las cuales se desempeña un egresado. En Educación estas competencias tienen relación con el conocimiento del contexto, la preparación de la enseñanza, la actuación pedagógica y la evaluación de los aprendizajes de sus alumnos y de su desempeño profesional.

En cuanto a quién evalúa y califica los aprendizajes de los estudiantes en la formación práctica se observan algunas diferencias entre las dos carreras. En Medicina los aprendizajes son evaluados y calificados por los tutores y supervisores directos, quienes están en conocimiento de los objetivos de aprendizaje y de evaluación. Además, existe una evaluación indirecta, ya que posteriormente este conocimiento práctico se utiliza como insumo en los cursos que siguen más tarde en el currículo.

En ambas carreras se evalúan estándares de desempeño, pero existen diferencias en los procedimientos e instrumentos utilizados. En Medicina se han incorporado progresivamente en estos últimos años pautas objetivas que evalúan las competencias a través de observación directa.

Otra modalidad que se emplea en la mayor parte de los cursos prácticos es el Examen Clínico Objetivo Estandarizado (OSCE), que permite explorar un amplio conjunto de 
habilidades y destrezas de una forma estandarizada, disminuyendo la variabilidad relacionada con la dificultad del problema y la evaluación por diferentes profesores. En los internados, una parte de la calificación es efectuada por la evaluación del desempeño del interno por el profesor, incluyendo el ámbito actitudinal.

En Educación los aprendizajes son evaluados y calificados tanto por el profesor colaborador en función del desempeño observado en el aula, como por el supervisor en función de las clases observadas y de los informes elaborados por los estudiantes con evidencias de las actividades realizadas y que son evaluadas, posteriormente, en base a rúbricas. Esta es la metodología de Muestras de Desempeño Docente (MDD) y que incluye la definición de estándares de logros, dimensiones y rúbricas para cada una de las dimensiones. Se atribuye un puntaje calificando el nivel de logro de acuerdo a los conceptos de insatisfactorio, básico, competente y destacado.

Por otra parte, se utilizan estrategias de autoevaluación, co-y heteroevaluación tanto de los procesos como de los resultados individuales y grupales. Se han definido algunos estándares de desempeño esperado, focos de atención e instrumentos a través del cual se verifican los resultados, los que tienen diferentes ponderaciones en la evaluación final.

\section{DISCUSIÓN Y CONCLUSIONES}

La formación de médicos y de profesores descansa, por un lado, en los conocimientos teóricos o disciplinares y, por otro, en el conocimiento profesional que se adquiere fundamentalmente a través de prácticas observando a médicos expertos y profesores con experiencia en sus contextos de desempeño.

Tanto en Medicina como en Educación la relación entre teoría y práctica no se reduce a la aplicación del conocimiento o de la teoría a la solución de un problema práctico. Además de esta relación se reconoce la especificidad del conocimiento profesional que se observa y aprende solo en contexto de práctica. El contexto de práctica y la interacción pedagógica que allí ocurre también es fuente de conocimientos y de preguntas que conducen a los estudiantes a nuevas preguntas e indagaciones teóricas. La práctica, a la vez, es una oportunidad para el desarrollo de la identidad profesional y para aprender la complejidad del oficio en un contexto concreto de desempeño.

En la Pontificia Universidad Católica de Chile (PUC) ambas carreras se definen a partir de un perfil de egreso que, además de compartir las definiciones comunes del proyecto educativo de la Universidad, se expresan en términos de competencias y estándares a lograr. Estas competencias, a su vez, tienen distinta relación con las agencias que regulan el campo de la profesión. En Medicina la definición de estándares nacionales y el control de su aprendizaje a través del EUNACOM tienen más trayectoria que la existente para el caso de Educación. Los estándares definidos en el 2001 y el 2011 por el Ministerio de Educación sólo son orientadores y el examen INICIA aplicado a los egresados de escuelas de educación aún es voluntario para los egresados de pedagogía.

En el currículo la experiencia práctica se concibe como progresiva definiendo oportunidades de aprendizaje práctico con distintos grados de autonomía según el nivel, situación problema y contexto de desempeño para ambas carreras. Sin embargo, en proporción del currículum en Medicina ocupa una parte más importante del tiempo de formación. 
Las principales diferencias que existen entre ambas carreras se establecen, en primer lugar, en el nivel de especificidad de las competencias de egreso; en segundo lugar, en el ámbito de la organización del dispositivo; en tercer lugar, en la articulación curricular y la evaluación de las prácticas.

A nivel de la especificidad de las competencias de los perfiles de egreso de ambas carreras, medicina presenta competencias con desempeños claramente establecidos tanto a nivel general como a nivel de cada especialidad en particular. En Educación, hasta el año 2011 no se contaba con competencias establecidas desde el Ministerio de Educación y las competencias de desempeño formuladas en el perfil de egreso son generales y no apuntan a dominios disciplinares particulares. Esto abre la discusión acerca del nivel de especificidad con que se pueden plantear las competencias en Educación, dado que parece primar una visión de la enseñanza como una actividad altamente dependiente del contexto y por lo tanto más dificil de especificar, a diferencia de la Medicina que se reconoce como una profesión con un alto nivel de estandarización.

En Medicina parece valorarse el estándar y el establecer patrones de desempeño efectivos para una determinada situación. En los documentos revisados en Educación aparecen menos desempeños sistematizados y especificados. Eso se traduce en que en medicina se presentan desempeños claves para los distintos ciclos de formación, lo que en Educación recién se está empezando a esbozar. Este es un núcleo que sería relevante explorar en futuras investigaciones, de tal manera que se pudiese avanzar en definir si la naturaleza de las profesiones lleva a que efectivamente una sea más estandarizable que la otra o si priman más bien variables de la cultura de la educación que se resisten a este tipo de especificaciones.

En Medicina existe una amplia tradición de campos clínicos donde estudiantes y médicos novicios realizan sus prácticas apoyadas por médicos docentes que, al mismo tiempo, ejercen la profesión en dicho campo. Estos docentes tienen una relación contractual con la Escuela de Medicina; su trabajo responde a un programa fuertemente regulado desde una coordinación central cuya tarea es garantizar la máxima consistencia interna entre los diferentes cursos de la malla curricular.

En Educación no existe un campo de práctica propio de la Facultad y por ello esta descansa en convenios con Establecimientos Educativos seleccionados según criterios de calidad y diversidad de dependencia. Los tutores directos de los estudiantes en prácticas son los profesores colaboradores, que no tienen una relación contractual con la Facultad de Educación, y los supervisores que son docentes universitarios que establecen la conexión entre la Facultad y los establecimientos y evalúan el desempeño de los estudiantes según los criterios definidos en los respectivos cursos de prácticas.

Estas relaciones entre los distintos actores podría ser un elemento que afecte la consistencia entre lo que se enseña en los cursos, los desempeños esperados en la práctica y las evaluaciones.

Existen además claras diferencias en cuanto a la organización y regulación entre los campos clínicos y los establecimientos educacionales que son centros de práctica. Los primeros están regulados por Decreto del Ministerio de Salud. Los segundos responden a un acuerdo establecido entre la Universidad y el Establecimiento educativo, donde la facultad tiene escasa injerencia en las actividades prácticas.

En ambas carreras intervienen distintos actores con diferentes jerarquías y responsabilidades en el proceso de formación. Sin embargo, la diferencia central es que el profesional 
tutor en Medicina es docente de la Escuela de Medicina. Tiene así una relación directa y contractual con la Institución académica responsable de la formación. En Educación se trata de una relación indirecta. El profesor colaborador, que es el tutor efectivo del estudiante en la sala de clases, tiene un estatus de voluntario con la institución académica. El estudiante de medicina puede observar a su profesor universitario como un profesional en ejercicio. En educación, en cambio, el estudiante observa a un profesor, elegido entre los mejores, pero que no es su docente ni se relaciona contractualmente con la Universidad.

Se puede inferir entonces una tradición diferente en cuanto a la articulación entre el saber académico propio de la facultad y el campo de la práctica en ambas carreras, que se sintetiza en la Tabla 2.

Tabla 2. Síntesis de diferencias y similitudes en la formación práctica de Medicina y Educación

\begin{tabular}{|c|c|c|}
\hline Dimensiones/Criterios & Medicina & Educación \\
\hline $\begin{array}{l}\text { Componentes de } \\
\text { formación }\end{array}$ & \multicolumn{2}{|c|}{$\begin{array}{l}\text { - Contemplan un área de formación práctica en contextos } \\
\text { clínicos y escolares como componente central del } \\
\text { currículo organizado en forma progresiva a lo largo de la } \\
\text { formación }\end{array}$} \\
\hline $\begin{array}{l}\text { Contexto del desempeño } \\
\text { profesional }\end{array}$ & $\begin{array}{c}\text { - Tradición alimentada por } \\
\text { la práctica e investigación } \\
\text { científica } \\
\text { - Implica una interacción } 1 \text { a } \\
1 \text { médico/paciente }\end{array}$ & $\begin{array}{l}\text { - Tradición alimentada } \\
\text { por la práctica distante de } \\
\text { investigación } \\
\text { - Implica una interacción } 1 \\
\text { a n profesor /alumnos }\end{array}$ \\
\hline $\begin{array}{l}\text { Definición de desempeños } \\
\text { en Perfil de egreso }\end{array}$ & $\begin{array}{l}\text { - precisos y protocolizados } \\
\text { y consensuados a nivel } \\
\text { nacional e internacional }\end{array}$ & $\begin{array}{l}\text { - Generales orientados } \\
\text { según estudios nacionales e } \\
\text { internacionales }\end{array}$ \\
\hline $\begin{array}{c}\text { Articulación del } \\
\text { componente de práctica }\end{array}$ & $\begin{array}{l}\text { - El currículo se articula en } \\
\text { torno a la formación práctica }\end{array}$ & $\begin{array}{l}\text { - Poca articulación de la } \\
\text { práctica con el currículo de } \\
\text { formación }\end{array}$ \\
\hline Itiner & $\begin{array}{l}\text { - La organización del } \\
\text { sistema permite que } \\
\text { todos los alumnos tengan } \\
\text { asegurada la rotación por } \\
\text { los diferentes campos } \\
\text { clínicos, en las diferentes } \\
\text { especialidades. }\end{array}$ & $\begin{array}{l}\text { - La organización del } \\
\text { sistema no garantiza que } \\
\text { un mismo estudiante tenga } \\
\text { prácticas en diferentes } \\
\text { contextos escolares. }\end{array}$ \\
\hline Campos de práctica & $\begin{array}{c}\bullet \text { Los hospitales tienen } \\
\text { tradición como campos } \\
\text { clínicos } \\
\text { - Las competencias } \\
\text { observadas son coherentes } \\
\text { con las competencias del } \\
\text { perfil de egreso }\end{array}$ & $\begin{array}{c}\text { - Las escuelas no tienen } \\
\text { tradición de centros de } \\
\text { práctica } \\
\text { • Los desempeños } \\
\text { observados no siempre } \\
\text { son coherentes con las } \\
\text { competencias del perfile de } \\
\text { egreso }\end{array}$ \\
\hline
\end{tabular}


En síntesis el análisis documental informa claramente sobre una mayor experiencia en la generación de contextos reales, pero controlados de enseñanza práctica de la medicina con vínculos explícitos y afianzados entre la facultad y el campo clínico. Esta alianza entre el campo de formación y el campo auténtico de trabajo plantea un desafío para las instituciones encargadas de la formación de profesores, en cuanto poder establecer o disponer espacios protegidos de calidad para los educadores en formación, asegurando la vinculación con modelos claves y estructurando un dispositivo que promueva el desarrollo de competencias propias del profesional de la educación. Una segunda distinción clave parece ser la dificultad de articulación de los ramos teóricos del currículo en la formación de profesores con los aprendizajes de conocimientos, habilidades y destrezas esperadas a ser desarrolladas en el campo práctico.

En futuros estudios incluiremos la visión de los actores para analizar cómo se perciben estas tensiones desde la experiencia práctica de estudiantes y docentes en los distintos niveles de la formación.

\section{REFERENCIAS BIBLIOGRÁFICAS}

Ávalos, B. (2002). Profesores para Chile. Historia de un proyecto. Santiago: Ministerio de Educación. Ávalos, B. y Aylwin, P. (2007). How young teachers experience their professional work in Chile. Teaching and Teacher Education, vol.23, n.4, 515-528

Babbie, E. (2000). Fundamentos de la investigación social. México: International Thomson.

Barber, M., \& Mourshed, M. (2008). Cómo hicieron los sistemas educativos con mejor desempeño del mundo para alcanzar sus objetivos. Santiago: Programa de Promoción de la Reforma Educativa en América Latina y el Caribe, Documento de trabajo n. 41.

Beraza, M. Z. (2011). El Practicum en la formación universitaria: estado de la cuestión. Revista de educación, $n$. 354, 21-43. Recuperado de http://dialnet.unirioja.es/servlet/articulo?codigo=3417760

Carraccio, C. L., Benson, B. J., Nixon, L. J., y Derstine, P. L. (2008). From the Educational Bench to the Clinical Bedside. Translating the Dreyfus Clinical Skills. Clinical Teaching, vol. 83, n.8, 761-767

Contreras, I., Rittershaussen, S., Montecinos, C., Solís, M. C., Núñez, C., \& Walker, H. (2010). Los centros educativos como espacios para aprender a enseñar. Visiones desde los programas de formación de profesores de educación media. Estudios Pedagógicos, vol. 36, n.1, 85-105.

Dent, J. A. (2005). Bedside teaching. En J. A. Dent \& R. M. Harden (Eds.), A practical guide for medical teachers (pp. 77-85). London: Elsevier

Darling-Hammond, L. (2006). Constructing 21st- Century Teacher Education. Journal of Teacher Education, vol. 57, n.3, 300-314.

Darling-Hammond, L. (2000). How Teacher Education Matters. Journal of Teacher Education, vol.51, n.3, 166-173.

Dreyfus, H. Dreyfus S. (1986). Mind over machine: the power of human intuition and expertise in the era of the computer. New York: The Free Press.

Enríquez, O. Mena, B. (2005). Habilitación profesional. Condiciones para el aseguramiento de la calidad de la educación médica y condiciones para la confianza recíproca. Experiencia y visión de ASOFAMECH. Revista Médica de Chile, n. 133, 483-494.

Facultad de Educación (2003). Planes de estudio conducentes a Licenciado en Educación y Profesor de Educación General Básica. Resolución de Vicerrectoría Académica $N^{\circ}$ 02/2003. Santiago: Pontificia Universidad Católica de Chile.

Grossman, P., Compton, C., Igra, D., \& Ronfeldt, M. (2011). Teaching Practice: A Cross-Professional Perspective 1. Teachers College Record, vol. 111, n.9, 2055-2100. 
Loo, S. (2007). Theories of Bernstein and Shulman: their relevance to teacher training courses in England using adult numeracy courses as an example. Journal of Further and Higher Education, vol. 31, n.3, 203-214

Marcelo García, C. (2007). La formación docente en la sociedad del conocimiento y la información: Avances y temas pendientes. Olhar de Profesor, vol. 10, n.1, 63-90. En: www.uepg.br/ olhardeprofesso (consultado 12 Diciembre 2012)

MINEDUC, (2001). Estándares de desempeño para la formación inicial docentes. Santiago: Ministerio de Educación de Chile

Munté, F. G., \& others. (2006). " Ojo clínico” y evidencia científica. Educación médica, vol. 9, n.1, 21-23. En: http://scielo.isciii.es/scielo.php?pid=S1575. (Consultado: 10 Agosto 2011)

Panel de Expertos, (2010). Propuestas para fortalecer la profesión docente en el sistema escolar chileno. Santiago; Ministerio de Educación de Chile.

Pérez-Gómez, A. (2010). Aprender a educar. Nuevos desafíos para la formación de docentes. Revista Interuniversitaria de Formación del Profesorado, vol. 68, n.242, 37-60.

Roget, D. À. (2009). La pratique réflexive dans le cadre du DPPE : recherche qualitative. Actes du 2eme colloque international francophone sur les méthodes qualitatives (pp. 1-12). En: www. trigone.univ-lille1.fr/cifmq2009 (Consultado 10 Noviembre 2012)

Schön, D. (1998). El profesional reflexivo: cómo piensan los profesionales cuando actúan. Barcelona: Paidós.

Shulman, L. S. (1998). Theory, Practice, and the Education of Professionals. The Elementary School Journal, vol. 98, n.5, 51-526

Strauss A. y J. Corbin (1990) Basics of qualitative research. Grounded theory procedures and techniques. London: Sage publications.

Zeichner, K. (2010). Rethinking the connections between campus courses and field experiencies in college-and university-based teacher education. Journal of Teacher Education, vol 61, n.1-2, 89-99. 